$\begin{array}{cl}\begin{array}{c}\text { Revue } \\ \text { de /histoire }\end{array} & \text { Revue de l'histoire des religions } \\ \text { des religions } & \begin{array}{l}3 \mid 2015 \\ \text { Varia }\end{array}\end{array}$

\title{
La crise du sacrement de pénitence dans le catholicisme français des années 1960-1970
}

The crisis of the sacrament of penance in the French Catholicism of the years 1960-1970

Guillaume Cuchet

\section{OpenEdition}

Journals

Édition électronique

URL : http://journals.openedition.org/rhr/8414

DOI : 10.4000/rhr.8414

ISSN : 2105-2573

Éditeur

Armand Colin

Édition imprimée

Date de publication : 1 septembre 2015

Pagination : 397-428

ISBN : 978-2-200-92993-0

ISSN : 0035-1423

\section{Référence électronique}

Guillaume Cuchet, «La crise du sacrement de pénitence dans le catholicisme français des années 1960-1970", Revue de l'histoire des religions [En ligne], 3 | 2015, mis en ligne le 01 septembre 2018, consulté le 19 avril 2019. URL : http://journals.openedition.org/rhr/8414 ; DOI : 10.4000/rhr.8414 


\section{La crise du sacrement de pénitence dans le catholicisme français des années 1960-1970}

La crise du sacrement de pénitence a été si profonde dans le catholicisme français des années 1960-1970 que certains s'imaginent qu'il a été tout bonnement "supprimé» par le concile Vatican II. Au début des années 1960 encore, son administration était considérée par le clergé comme l'une des charges les plus chronophages du ministère pastoral. La crise de la confession est, de ce point de vue, l'un des aspects les plus révélateurs de la "crise catholique» des années 1965-1975. Elle constitue un fait sociologique et spirituel majeur qui incite à revenir sur la conjoncture particulière des années 1960-1970, pour essayer de comprendre ce qui a pu précipiter cette chute et voir ce qu'elle peut nous apprendre sur l'histoire religieuse et culturelle de la période elle-même.

The crisis of the sacrament of penance in the French Catholicism of the years 1960-1970

The crisis of the sacrament of penance was so deep in the French Catholicism of the years 1960-1970 that some people imagine nowadays that it was plainly "abolished" by the council Vatican II. At the beginning of the sixties still, its administration was considered by the clergy as one of the most chronophage duties of the pastoral ministry. The crisis of the confession is, from this point of view, one of the most revealing aspects of the "catholic crisis" of the years 1965-1975. It constitutes a major sociological and spiritual fact which incites to come back to the peculiar situation of the years 1960-1970 to try to understand what might have accelerate this fall and to see what it can teach us about the religious and cultural history of the period itself. 
Un certain nombre de faits récents, notamment des publications ${ }^{1}$, certaines déclarations du pape François, des initiatives pastorales du type «journées du pardon», des remises en service de confessionnaux parfois ${ }^{2}$, donnent à penser que pourrait bien se dessiner dans le catholicisme contemporain un mouvement de redécouverte de la confession ou, en termes techniques, du sacrement de la pénitence et de la réconciliation. L'avenir dira ce qu'il faut en penser, notamment s'il ne s'agit que d'une manifestation parmi d'autres du mouvement de retour à certains usages préconciliaires (de la soutane au latin) qui prévaut actuellement dans une partie du catholicisme français, ou d'un mouvement plus profond. Mais tel quel, il fournit d'ores et déjà à l'historien (qui ne sait jamais très bien de quoi il est le symptôme) l'occasion de revenir sur la crise d'un sacrement qui fut, dans les années 1960-1970, si brutale et si profonde que certains ont pu s'imaginer qu'il avait été tout bonnement «supprimé» par le concile Vatican II (1962-1965).

De fait, quiconque fréquente ou visite un tant soit peu les églises n'a pu manquer d'être frappé un jour par les files de confessionnaux désaffectés qui peuplent les bas-côtés de bon nombre d'entre elles, surtout si elles sont de taille importante. Ils font figure de vestiges, plus ou moins décoratifs, d'un ancien régime pénitentiel révolu autant que de flux largement taris, mais dont on devine qu'ils ont dû être très importants. Difficile de se figurer aujourd' hui, en effet, qu' au début des années 1960 encore, avec des nuances importantes selon les régions, les confessionnaux étaient pris d'assaut à la veille des grandes fêtes religieuses et que le sacrement de pénitence était considéré par le clergé comme l'un des plus chronophages du ministère pastoral, avec les enterrements. Il suffit de se rendre (sans trop tarder il est vrai) dans une maison de retraite du clergé, de préférence dans une ancienne région de «chrétienté», pour, en discutant avec les pensionnaires, se

1. Par exemple: «Ils veulent revivifier la confession», dans La Croix du 28 février 2014, et l'ouvrage dirigé par Hélène Bricout et Patrick Prétot, Faire pénitence. Se laisser réconcilier. Le sacrement comme chemin de prière, Paris, Le Cerf, coll. «Lex Orandi», 2013, rapidement épuisé.

2. Par exemple, Michel-Marie Zanotti-Sorkine, Au diable la tiédeur. Suivi d'un Petit traité de l'essentiel, Paris, Robert Laffont, 2012, p. 66. 
rendre compte de ce qu'était cette confession de masse des années 1950, devenue pour nous si exotique ${ }^{3}$.

La crise de la confession est, de ce point de vue, l'un des aspects les plus révélateurs et les plus frappants de ce qu'il est désormais convenu d'appeler, dans l'historiographie, la «crise catholique» des années 1965-1975. Dans un dossier du Pèlerin du 3 novembre 1974, intitulé «Les Français et la confession. Un sondage SOFRESPèlerin », un aumônier d'Action catholique, supérieur de grand séminaire, écrivait: «Dans l'Église, la confession, cela a été la chute libre sans parachute. Cette chute n'a été rencontrée nulle part ailleurs, ni pour l'eucharistie ni pour la foi $»^{5}$, comme si elle avait rajouté à la crise générale les effets de sa crise particulière de longue durée. L'évolution de la doctrine officielle du sujet, qui a vu succéder à l'ancien «tribunal de la pénitence» le nouveau, et a priori plus engageant, «sacrement de la réconciliation», n'y a rien changé, pas plus que l'abandon des confessionnaux ou la diversification des formes de la pénitence.

Cette chute de la confession constitue donc en soi un fait sociologique et spirituel majeur dont historiens et sociologues n'ont sans doute pas pris toute la mesure: rien moins, en somme, que la foudroyante mutation par abandon, en l'espace de quelques années seulement, d'une pratique qui a profondément façonné les mentalités catholiques dans la longue durée, ainsi que les formes culturelles de la culpabilité individuelle et collective ${ }^{6}$. Le phénomène incite à

3. Je me réfère ici aux échanges que j'ai eus en janvier 2013, grâce aux bons soins du père Henri Baudry, ancien archiviste du diocèse de Luçon, avec des pensionnaires de la maison de retraite du clergé du Landreau aux Herbiers (Vendée), notamment les pères Auguste Roy $(\dagger)$, André Baudon ( $†$ ), Jean Grelier, Jacques David, Alphonse Drié, Marcel Maingourd et Pierre Hervouet. Qu'ils en soient tous chaleureusement remerciés.

4. Denis Pelletier, La crise catholique. Religion, société, politique, Paris, Payot, 2002.

5. «Les Français et la confession. Un sondage SOFRES-Pèlerin», dans Le Pèlerin du XX siècle, 3 novembre 1974, p. 41. L'auteur était prêtre depuis quinze ans et avait donc été ordonné à la fin des années 1950.

6. Le phénomène n'a pas encore fait l'objet d'une étude historique approfondie. L'historiographie française de la confession à l'époque contemporaine a été marquée par trois ouvrages majeurs, ceux de Jean Guerber, Le ralliement du clergé français à la morale liguorienne. L'abbé Gousset et ses précurseurs (1785-1832), Rome, Université Grégorienne, coll. «Analecta Gregoriana», 1973; l'ouvrage collectif du Groupe de la Bussière, Pratiques de la confession. Des pères du désert à Vatican II. Quinze études d'histoire, Paris, Le Cerf, 1983; et Jean Delumeau, L'aveu et le pardon. Les difficultés de la confession XIII'-XVIII' siècle, Paris, Fayard, 1990. 
revenir sur la conjoncture particulière des années 1960-1970, pour essayer de comprendre ce qui a pu précipiter cette chute et voir ce qu'elle peut nous apprendre sur l'histoire religieuse et culturelle de la période.

\section{LA CHUTE DE LA CONFESSION, UN FAIT SOCIOLOGIQUE ET SPIRITUEL MAJEUR}

\section{L'ampleur statistique du décrochage}

La confession n'a guère retenu l'attention de la sociologie religieuse du XX $\mathrm{X}^{\mathrm{e}}$ siècle en France, ni dans sa version pastorale classique, autour de Fernand Boulard et de ses disciples, ni dans sa version laïque et universitaire. Tout au plus la première paraît-elle avoir croisé le problème à propos de ceux qu'elle appelait, dans son jargon, les «cénalisants» (c'est-à-dire les fidèles qui communient souvent), et de l'inversion, au tournant des $\mathrm{XIX}^{\mathrm{e}}$ et $\mathrm{XX}^{\mathrm{e}}$ siècles, des courbes de la pratique dominicale et pascale qui constituaient ses indices phares. $\mathrm{Au} \mathrm{XIX}^{\mathrm{e}}$ siècle, en effet, l'assistance à la messe dominicale était généralement supérieure à la pratique pascale parce que celle-ci représentait un geste plus onéreux et plus personnel, dans la mesure où (précisément) son accès était conditionné par la confession. Or au $\mathrm{XX}^{\mathrm{e}}$ siècle, sans qu'on sache encore très bien pourquoi, la situation s'est inversée, à des dates variables selon les diocèses?

Pour autant, les données quantitatives ne sont pas totalement absentes dans ce domaine et l'on dispose sur l'évolution de la confession à l'époque contemporaine d'une série éclairante de trois sondages qui fournissent des données comparables pour les années 1952, 1974 et $1983^{8}$.

Le premier a été réalisé par l'Institut français d'opinion publique (IFOP) à la demande de la revue Réalités en 1952. Il faisait partie

7. Voir les remarques de Claude Langlois dans «"Toujours plus pratiquantes". La permanence du dimorphisme sexuel dans le catholicisme français contemporain», dans Clio. Histoire, Femmes et Sociétés, $\mathrm{n}^{\circ}$ «Femmes et Religions », 2-1995, p. 229-260.

8. Les données proviennent du livre de Jacques Sutter, La vie religieuse des Français à travers les sondages d'opinion (1944-1976), t. 2, Paris, CNRS, 1984, p. 898-901, mais le tableau comparatif a été réalisé par le sociologue Julien Potel dans : «La Pénitence», dans La Maison-Dieu, 1986, p. 44-45. 
d'une enquête plus large sur l'état du catholicisme français qui a suscité tout un débat à l'époque parce qu'elle montrait, contre les diagnostics pessimistes du mouvement «missionnaire» d'aprèsguerre $^{9}$, qu'il était encore très consistant socialement et qu'il n'avait nul besoin des thérapies de choc que certains voulaient lui appliquer. Le deuxième, réalisé par la SOFRES en septembre 1974 et publié dans un numéro du Pèlerin de novembre ${ }^{10}$, correspondait à la publication en février précédent du nouveau rituel romain de la pénitence, annoncé dix ans plus tôt par le concile Vatican II $^{11}$. Le troisième, réalisé également par la SOFRES en février 1983 et publié dans un numéro du Pèlerin d'avril ${ }^{12}$, était lié à la parution en janvier du nouveau Code de droit canonique de Jean-Paul II et aux discussions sur les «célébrations pénitentielles» avec absolution collective, autorisées par le nouveau rituel mais que Rome s'efforçait de raréfier. Les deux tableaux suivants, empruntés à un article important de Julien Potel de 1986 sur le sujet, résument les principaux enseignements statistiques des trois enquêtes.

Tableau $\mathrm{n}^{\circ} 1$ : évolution de la fréquence de la confession parmi les catholiques français

\begin{tabular}{|l|c|c|c|}
\hline Fréquence & $\mathbf{1 9 5 2}$ & $\mathbf{1 9 7 4}$ & $\mathbf{1 9 8 3}$ \\
\hline Au moins une fois par mois & 15 & 1 & 1 \\
\hline Quelques fois par an & 18 & 16 & 8 \\
\hline Une fois par an & 18 & 12 & 5 \\
\hline Au moins une fois par an & 51 & 29 & 14 \\
\hline Moins d'une fois par an & 9 & 13 & 13 \\
\hline Jamais & 37 & 54 & 69 \\
\hline Non déterminé & 3 & 4 & 4 \\
\hline Total & 100 & 100 & 100 \\
\hline
\end{tabular}

9. Représenté notamment par France, pays de mission? (1943) des abbés Godin et Daniel, ou Feu la chrétienté (1950) du philosophe Emmanuel Mounier.

10. Le Pèlerin, $\mathrm{n}^{\circ} 4797,3$ novembre 1974.

11. La version latine (Ordo paenitentiae) est datée du 2 décembre 1973 mais elle n'a été rendue publique qu'en février 1974. La version francophone officielle date de 1978, avec un titre différent, indice d'inflexions théologiques et pastorales assez sensibles: Célébrer la pénitence et la réconciliation. Nouveau rituel, Chalet-Tardy.

12. Le Pèlerin, $\mathrm{n}^{\circ} 5235,3$ avril 1983. 
Tableau $\mathrm{n}^{\circ} 2$ : confession et différences de genre

\begin{tabular}{|l|c|c|c|c|}
\hline Fréquence & $\begin{array}{c}\text { Hommes } \\
\mathbf{1 9 5 2}\end{array}$ & $\begin{array}{c}\text { Hommes } \\
\mathbf{1 9 7 4}\end{array}$ & $\begin{array}{c}\text { Femmes } \\
\mathbf{1 9 5 2}\end{array}$ & $\begin{array}{c}\text { Femmes } \\
\mathbf{1 9 7 4}\end{array}$ \\
\hline $\begin{array}{l}\text { Au moins une fois } \\
\text { par mois }\end{array}$ & 7 & 1 & 23 & 1 \\
\hline Quelques fois par an & 15 & 11 & 21 & 21 \\
\hline Une fois par an & 18 & 10 & 18 & 13 \\
\hline Au moins une fois par an & 40 & 22 & 62 & 45 \\
\hline Moins d'une fois par an & 8 & 11 & 9 & 14 \\
\hline Jamais & 49 & 62 & 26 & 47 \\
\hline Non déterminé & 3 & 5 & 3 & 4 \\
\hline Total & 100 & 100 & 100 & 100 \\
\hline
\end{tabular}

En 1952, plus de la moitié (51\%) des adultes catholiques déclaraient se confesser au moins une fois par an, généralement à Pâques, comme il était d'obligation de le faire depuis le canon vingt et un du quatrième concile du Latran de 1215, confirmé par le concile de Trente au XVI ${ }^{e}$ siècle. Sans doute faut-il faire la part dans l'interprétation de ce résultat d'une probable «surdéclaration» du niveau de pratique de la part des personnes interrogées, comme le grief en avait été formulé à l'époque contre l'ensemble de l'enquête. Mais, outre le fait que celle-ci était déjà en soi significative (elle a disparu ensuite), même ainsi corrigé, le niveau de conformité présumé restait élevé. Manifestement, au lendemain de la Seconde Guerre mondiale, la confession n'était plus tout à fait l'obstacle qu'elle avait été par le passé, au XIX ${ }^{\mathrm{e}}$ siècle notamment, surtout pour les hommes. Boulard, dans son commentaire de l'enquête de 1952, avait bien noté cette apparente banalisation: «[à] l'occasion du passage de Notre-Dame de Boulogne, écrivait-il, d'une mission ou d'un pèlerinage, le Français moyen ne répugne peut-être plus à aller jusqu'à la confession. [...] Autrefois, la confession - acte vraiment personnel et souvent barrière importante à franchir - était le signe d'une conversion, d'un changement de vie. Aujourd'hui, finirait-elle, pour le Français moyen, par faire partie du "conformisme saisonnier"? $\gg^{13}$. On peut penser

13. «Un pays où 94 pour 100 des citoyens sont baptisés et où 43 pour 100 des adultes font leurs Pâques est-il un pays de mission? Une mise au point de M. le chanoine Boulard», La Croix, 4-5 janvier 1953, p. 6. 
qu'ont pu jouer en ce sens la baisse de l'anticléricalisme, la quasidisparition du délai d'absolution (encore en usage, dans certains cas, au début du siècle) et donc de la possibilité d'être éconduit, une plus grande mobilité de la population et la démultiplication corrélative des possibilités de confession extraordinaire et la multiplication des missions d'après-guerre ${ }^{14}$.

À l'intérieur de ce groupe conséquent de $51 \%$ de pénitents en règle, ou qui, du moins, se prétendaient tels, se détachait un sousgroupe d'environ $15 \%$ de pénitents fréquents, qui se confessaient une fois par mois, dont $2 \%$ le faisaient même toutes les semaines. Ces «virtuoses» parmi les «dévots» (pour parler le langage des sociologues) représentaient plusieurs dizaines de milliers de personnes, soit beaucoup plus que les seuls religieux, religieuses et séminaristes qui y étaient astreints canoniquement ${ }^{15}$.

Au total, la confession des années 1950 se présentait donc sous une double figure: d'une part, un flux intermittent de pénitents occasionnels, lié aux grandes fêtes du calendrier liturgique, qui culminait le samedi saint, à la veille de Pâques; d'autre part, un flux permanent de pénitents fréquents, qui se confessaient toutes les semaines ou tous les mois, parce qu'ils avaient l'âme particulièrement «délicate» (comme on disait au XIX ${ }^{\mathrm{e}}$ siècle) ou qu'ils pratiquaient une confession «de dévotion», comme le recommandait encore Pie XII dans son encyclique Mystici corporis en 1943. Le principe, contesté par personne, était que plus on était chrétien, plus on se confessait. Si l'on ajoute l'importante «clientèle captive» des enfants du catéchisme et des écoles catholiques, celle des collèges, des petits séminaires et des maisons d'éducation religieuse, encore accrue par le baby-boom (les églises des années 1950 étaient pleines d'enfants et de jeunes), on voit que la pratique pénitentielle représentait une part importante de l'activité pastorale du clergé.

En 1974, la situation était bien différente. Les catholiques n'étaient plus que $29 \%$ à se confesser au moins une fois par an, soit pas loin d'une division par deux du total de 1952, et - phénomène peut-être plus spectaculaire encore - le groupe des pénitents fréquents avait

14. L'après-guerre est une grande époque de missions, comparable, mutatis mutandis, à celle de la Restauration (1815-1830), sous l'égide du Centre pastoral des missions de l'intérieur.

15. Voir les canons 595, 1367 et 125 du Code de 1917. 
quasiment disparu, passant de 15 à $1 \%$. En 1983, non seulement ces derniers n'avaient pas reparu mais la proportion des pénitents simplement en règle était passée de 29 à $14 \%$, le tout à obligations canoniques constantes ${ }^{16}$.

Ces statistiques ont quelque chose de fascinant pour le sociologue ou l'historien, et l'on peut s'étonner qu'elles n'aient pas davantage retenu l'attention. Elles permettent d'ores et déjà de formuler quelques constats élémentaires.

En premier lieu, plus que d'évolution de la pratique, il convient de parler de véritable mutation, avec tout ce que le terme suggère de brutalité dans la rupture, et même de mutation par abandon, dans la mesure où tous les niveaux de pratique sont concernés par le déclin. On ne constate pas de phénomène de récupération dans les étages inférieurs de ce qui a été perdu au niveau supérieur, hormis (et encore très légèrement) dans la catégorie de ceux qui déclaraient se confesser «moins d'une fois par an». Il ne s'agit donc pas de la recomposition d'un système, ni même de son relâchement, mais bien de sa «décomposition», pour reprendre le titre d'un essai célèbre de Louis Bouyer de $1968^{17}$.

En deuxième lieu, s'impose comme un fait majeur la disparition entre 1952 et 1974 du groupe des pénitents fréquents, c'est-àdire de ces quelque $15 \%$ de catholiques qui disaient se confesser au moins une fois par mois et qui représentaient la partie la plus institutionnellement intégrée des fidèles, celle qui s'efforçait d'articuler avec le plus de cohérence, pratiques, croyances et comportements. Ils constituaient le cœur de l'Église et sa véritable force de frappe sociale. Or ils se sont comme volatilisés entre les deux dates, comme si le noyau dur du catholicisme français avait «éclaté», pour reprendre le titre d'un livre célèbre de Michel de Certeau et Jean-Marie Domenach paru en 1974' ${ }^{18}$.

Cet éclatement signifie que ce sont, pour une bonne part, des émancipés de fraîche date de la pénitence traditionnelle - d'aucuns diraient des échappés récents de confessionnaux - qui ont «mis en

16. Voir le canon 988, \& 1 du Code de 1983, où l'obligation annuelle est rappelée.

17. Louis Bouyer, La décomposition $d u$ catholicisme, Paris, AubierMontaigne, 1968.

18. Michel de Certeau, Jean-Marie Domenach, Le christianisme éclaté, Paris, Le Seuil, 1974. 
crise $»^{19} l^{\prime}$ institution dans les années 1960 . Il est difficile de ne pas faire le rapprochement ici avec le développement de la contestation interne dans l'Église, la transgression des règles et tabous hier encore en vigueur et la mise en accusation de l'institution, celle-là même, pensait-on, qui avait imposé si longtemps, sans profit apparent, de si lourdes obligations. Il y aurait un rapprochement à faire avec le tableau du «récidiviste découragé» dans la littérature pénitentielle de l'âge classique, traditionnellement décrit comme en colère contre l'institution et tenté, pour rattraper le temps perdu, par toutes les transgressions. Le lien a été souligné, de façon polémique, par ce converti «contracyclique» qu'était Maurice Clavel dans un ouvrage célèbre de 1976: «Accusons-nous, ou nous culpabiliserons par névrose! Battons nos coulpes ou nous aurons des flagellants! Confessons-nous ou nous aurons des convulsionnaires !... Et surtout n'allez pas plaider coupable pour toute l'Église depuis deux siècles et par jeu de bascule décréter l'innocence de toute l'Humanité, perdue depuis toujours par l'échange égoïste et récupérée d'un coup par la collectivisation des machines, ou des coïts, ou des deux... Allez, c'est plus obscur et noueux que ça, l'homme, même sans Dieu $\gg^{20}$.

Le dernier constat concerne les femmes. En 1952, parmi les catholiques qui se confessaient au moins une fois par mois, on comptait $7 \%$ des hommes et $23 \%$ des femmes (un ratio de un à trois qui n'était, somme toute, pas si déséquilibré que cela). Sans surprise, on retrouvait dans ce domaine, la trace du «dimorphisme sexuel» de la pratique religieuse, d'autant plus que les réactions différenciées face à la confession ont souvent été décrites par les historiens comme étant à l'origine du clivage lui-même ${ }^{21}$. En 1974 cependant, l'écart a disparu: les deux sexes ont atteint ensemble le plancher de la pratique à $1 \%$, ce qui signifie pour les femmes un effondrement d'autant plus spectaculaire qu'il est sans véritable équivalent ailleurs, dans les autres indices de la sociologie

19. J'emprunte cette notion de «mise en crise» à Yann Raison du Cleuziou qui l'emploie dans son bel article: «Albert-Marie Besnard, un maître dépassé par ses disciples. Mystique de la génération et subversion des formes de l'autorité au couvent d'étude dominicain au Saulchoir», dans Genèses, 2012/3, n 88, p. 25-45.

20. Maurice Clavel, «Dieu est Dieu, nom de Dieu!», Paris, Grasset, 1976, p. $144-145$.

21. Voir notamment l'article de Ralph Gibson: «Le catholicisme et les femmes en France au XIX ${ }^{\mathrm{e}}$ siècle», dans Revue d'histoire de l'Eglise de France, LXXIX, $\mathrm{n}^{\circ} 202$, janvier-juin 1993, p. 69-93. 
religieuse, notamment au niveau de la pratique dominicale, où les anciens contrastes de genre se sont beaucoup mieux conservés.

Au total, la crise du sacrement de pénitence paraît donc avoir été double: d'une part, la quasi-disparition du groupe des pénitents fréquents selon une chronologie qui reste à préciser (en 1972, la Congrégation pour la doctrine de la foi rappelle au clergé qu'il ne faut pas déconsidérer la confession «de dévotion $»^{22}$ ); d'autre part, le tarissement partiel et plus progressif du flux des pénitents ordinaires.

\section{Le tournant du milieu des années 1960}

Notre source ne nous dit pas, malheureusement, quand exactement a eu lieu la rupture, de sorte que, sur ce point, nous en sommes un peu réduits aux conjectures et aux hypothèses. En 1974 en tout cas, le fait paraissait déjà acquis depuis un certain temps. À propos de la parution du livre de Jacques Durandeaux, Chrétiens au feu de la psychanalyse (1972), le dominicain Jean-Claude Sagne écrivait: «La forme actuelle du sacrement de pénitence subit une désaffection trop générale et trop profonde pour que [cet] essai [...] puisse vraiment alourdir le dossier d'une cause en fait déjà entendue $»^{23}$.

À la fin des années 1950 et au début des années 1960, il était déjà question dans certaines publications religieuses, comme la revue de liturgie La Maison-Dieu ou de spiritualité jésuite Christus, de «crise du sacrement de pénitence». Mais il est assez difficile de savoir si cette «crise» était dans le sacrement lui-même, dont la pastorale était, en tout état de cause, problématique depuis au moins les lendemains de la Révolution, ou dans le regard théologique et pastoral porté sur lui par les milieux avancés du catholicisme français qui s'exprimaient dans ces organes. Bien souvent, en réalité, il s'agissait de désigner par cette expression la multiplication des cas de communion sans confession, qui attestaient moins d'un déclin de la pénitence elle-même que de la diffusion de la communion fréquente, ou la tendance, dans une partie du jeune clergé, à déconsidérer la pratique de la confession fréquente recommandée

22. Congrégation pour la Doctrine de la foi, «Normes pastorales pour l'administration de l'absolution sacramentelle générale», dans La Documentation catholique, 6-20 août 1972, t. LXIX, nº 1614, p. 715.

23. Jean-Claude Sagne, o.p., «Le procès de l'aveu», dans La Maison-Dieu, 117, 1974, p. 137. 
par Pie XII. De même, dans la littérature assez abondante consacrée, au tournant des années 1950-1960, à la question de la «révision de vie $»^{24}$ au sein de l'Action catholique et au-delà, il n'était pas question de chute parallèle de la confession, point qu'on n'aurait pas manqué de signaler le cas échéant, dans la mesure où l'un des enjeux doctrinaux était de bien distinguer les deux.

Donc si la rupture n'a pas encore eu lieu au début des années 1960, c'est qu'elle a eu lieu après, probablement au milieu de la décennie. Plusieurs éléments plaident en ce sens. Le premier est que c'est aussi à ce moment-là que fléchissent nettement les courbes de pratique dominicale ${ }^{25}$ et qu'il y a des chances pour qu'il y ait dans ce domaine une solidarité des indices. Le second est qu' une enquête de décembre 1967 montrait que $25 \%$ des catholiques s'étaient confessés depuis la dernière fête de Pâques et $8 \%$ seulement depuis un mois $^{26}$, ce qui incite à penser que la situation de 1974 , notamment la disparition du groupe des pénitents fréquents, était déjà en cours d'acquisition. Indice supplémentaire, même s'il est plus qualitatif: la place que tient la crise de la confession dans ce texte emblématique de la «crise catholique» qu'est «Le troisième homme» de François Roustang, paru en 1966 dans Christus. Au moment de donner figure concrète à ces chrétiens qui s'éloignaient silencieusement de l'Église, Roustang mentionnait (même si l'on ne sait pas s'il s'agit d'une personne réelle ou simplement d'un type artificiellement créé pour les besoins de sa démonstration) un «pilier de l'Action catholique depuis plus de vingt ans» qui avait décidé récemment d'arrêter de se confesser "parce que ça ne servait à rien », et qui ne s'en portait pas plus $\mathrm{mal}^{27}$.

24. Voir notamment l'ouvrage de synthèse de Jourdain Bonduelle, La révision de vie. Situation actuelle, Paris, Le Cerf, 1964, qui reprend des articles du début des années 1960.

25. Je me permets de renvoyer ici à mon article: «Le dernier problème de Fernand Boulard: la rupture de pente religieuse de 1965», repris dans Faire de l'histoire religieuse dans une société sortie de la religion, Paris, Publications de la Sorbonne, coll. «Itinéraires», p. 103-139.

26. Jacques Sutter, La vie religieuse des Français à travers les sondages d'opinion (1944-1976), op. cit., p. 898. Les résultats de cette enquête ont été publiés dans la Vie catholique illustrée, no 1182 , 3-9 avril 1968.

27. François Roustang, s.j., «Le troisième homme», dans Christus, $\mathrm{n}^{\circ}$ 52, t.13, octobre 1966, p. 562. À rapprocher, pour mesurer son évolution personnelle en quelques années, de sa défense et illustration de la confession dans: "Pénitence et liberté», dans Christus, n 12, octobre 1956, p. 497-506. 
On pourrait donc, sous réserve d'une enquête plus approfondie, résumer la séquence globale de la manière suivante: crise larvée de la confession fréquente dès la fin des années 1950, crise ouverte de la confession en général à partir du milieu des années 1960, amplification de la rupture après 1968. Dans tous les cas, la publication du nouveau rituel romain de la pénitence en février 1974 est intervenue trop tardivement pour enrayer la chute.

\section{LeS CAUSES DE LA CRISE}

Constater un phénomène est une chose, l'expliquer en est largement une autre. L'objectif de cette deuxième partie sera de faire l'inventaire des causes possibles de cette rupture, en essayant de préciser quel rôle elles ont joué dans le processus global, étant bien entendu qu'il est généralement difficile de faire le départ en elles entre ce qui relève de la cause et de la conséquence.

\section{Un facteur déclencheur:}

\section{la sortie de la culture de la pratique obligatoire}

La première raison tient à la sortie de la culture de la pratique obligatoire sous peine de péché mortel, si longtemps prévalente en catholicisme, même si le renouveau des années 1930-1960, notamment au sein de l'Action catholique, avait déjà commencé à déplacer les accents pour une partie des fidèles, surtout parmi les jeunes et les militants. La confession était le régulateur par excellence de cette très ancienne culture, jusque dans ses aspects les plus techniques, comme l'observation des règles du jeûne eucharistique (assoupli après la guerre) et les dispenses de messe dominicale, dont beaucoup de Français d'un certain âge ont gardé un vif souvenir dans lequel, bien souvent, un certain amusement le dispute à l'incrédulité rétrospective. C'est un phénomène fondamental, qui fait sentir partout ses effets dans notre documentation, mais qui reste difficile à saisir parce qu'il ne correspond à aucune consigne officielle, ni conciliaire, ni pontificale, ni épiscopale. Il relève surtout des effets d'ébranlement indirects du concile.

Le fait est que l'on assiste alors à une dépénalisation tout à fait nouvelle de l'abstention religieuse, considérée désormais comme 
secondaire au regard des «vrais» critères de christianisme que seraient la sincérité des consciences, l'engagement militant, le service de la cité et des pauvres, etc. Les pratiques religieuses ont cessé alors d'être présentées comme des «devoirs» impérieux - ce qu'on appelait dans les anciens catéchismes les «commandements de Dieu » et «de l'Église»-, dont il fallait s'acquitter, bon gré mal gré, parce que c'était l'habitude, que le clergé le demandait et qu'il en allait du salut. Dans l'ancien système, on n'imaginait certes pas que la courbe des pratiquants correspondît nécessairement à celle des élus dans l'au-delà, mais on ne doutait pas qu'il dût y avoir malgré tout un rapport entre les deux ${ }^{28}$. Désormais, la pratique religieuse devait être d'abord volontaire (ne venait-on pas de consacrer tardivement la liberté de conscience ?) et, à la limite, mieux valait s'abstenir si l'on ne s'en acquittait que pour de «basses» raisons, comme d'obéir à de vieilles habitudes ou par crainte de Dieu.

Il en est résulté un vaste phénomène de décompression collective, que l'on pourrait comparer, mutatis mutandis, au choc induit par la Révolution française à la fin du XVIII ${ }^{\mathrm{e}}$ siècle quand les pratiques ont cessé d'être civilement obligatoires pour ne plus l'être que canoniquement et pastoralement. L'observance de toutes les pratiques s'en est ressentie mais à plus forte raison celle de la confession, qui avait toujours été la plus coûteuse et la plus problématique pour le grand nombre.

\section{Le silence sur les «fins dernières»}

La deuxième raison, qui n'est pas sans lien avec la précédente, tient aux changements survenus dans la conception du salut. La peur de l'enfer - Jean Delumeau l'a souligné dans des études classiques - a été, pendant longtemps, l'un des principaux moteurs de la confession. Le motif était considéré comme normal par le clergé,

28. À titre d'exemple, le sociologue jésuite Émile Pin, dans sa remarquable monographie sur la paroisse de Saint-Pothin à Lyon dans les années 1950, écrivait dans son introduction: «Ce geste [l'assistance dominicale] est, pour un catholique, obligatoire sous peine de faute grave. C'est-à-dire qu'aux yeux de l'Église catholique le refuser consciemment, sans raisons sérieuses, fait perdre la grâce sanctifiante, rompt l'amitié avec Dieu, compromet le salut de l'âme, si le repentir par la contrition parfaite ou le sacrement de pénitence ne vient rétablir l'homme dans l'amitié divine.» (Pratique religieuse et classes sociales dans une paroisse urbaine, Saint-Pothin à Lyon, Paris, Spes, 1956, p. 8). 
même si des raisons plus positives, comme l'amour désintéressé de Dieu, étaient jugées préférables. La théologie morale faisait bien la différence entre l' «attrition» et la «contrition», ou la «crainte filiale» et la «crainte servile», mais elle considérait qu'à tout prendre, il valait mieux aller à Dieu par la voie de la peur que de ne pas y aller du tout. Dans le manuel de l'abbé Chanson, dont la première édition date de 1948 et qui a été largement diffusé dans les séminaires et les presbytères français des années 1950, il était encore bien précisé que la croyance en l'existence du «Dieu rémunérateur» («qui récompense les bons au Ciel et punit les méchants en Enfer») était « de nécessité de moyen» pour être sauvé. Dans les régions de «chrétienté» de la «carte Boulard», l'enfer, le purgatoire, le jugement, la mort subite, etc., faisaient encore partie de la pastorale ordinaire, a fortiori de celle des missions, même si les choses avaient d'ores et déjà commencé à évoluer dans les jeunes générations sacerdotales et militantes.

Or le clergé a cessé assez brutalement de parler de tous ces sujets autrefois si délicats, comme s'il avait cessé d'y croire lui-même, en même temps que triomphait dans le discours une nouvelle vision de Dieu, de type plus ou moins rousseauiste: le «Dieu Amour» (et non plus seulement «d'amour») des années 1960-1970. Le même Delumeau parlait, pour désigner le phénomène, de la «révolution spirituelle de notre temps». Une partie de ceux qui avaient été formés dans l'ancien catholicisme et qui s'en accommodaient ont pu se sentir déstabilisés par le changement ${ }^{29}$ : «les curés ont goudronné la route du ciel», disait au sociologue Fanch Elégoët une vieille paysanne bretonne, au début des années $1970^{30}$. Moyennant quoi, s'il n'y avait plus de péché ni d'enfer, du moins de péché un peu sérieux (susceptible de vous priver du ciel), l'utilité de la confession, dans sa définition traditionnelle, était effectivement moins évidente.

29. Voir le compte rendu d'une réunion préparatoire à une mission dans le canton de Saint-Fulgent en Vendée en 1970, p. 140-141 notamment: «Quelqu'un pense qu'aujourd'hui, après le concile, il n'y a plus de péché»; "Il semble qu'actuellement, il n'y ait plus de péché. On ne sait pas où est la limite entre le péché minime et le péché grave. La messe est-elle obligatoire?»; [Autrefois] on nous faisait voir le péché partout, nous étions damnés au feu de l'enfer. Ce qui gêne beaucoup, vue l'évolution actuelle, les personnes qui ont entendu ces choses-là ».

30. Formule citée par Yvon Tranvouez dans une lettre qu'il m'a adressée le 2 mars 2014. 


\section{La déconnexion entre confession et communion}

Une autre raison a joué un rôle fondamental dans la déprise: la déconnexion croissante entre confession et communion, amorcée dans les années 1950 et généralisée dans les années 1970. Une partie du clergé contemporain tente, avec un succès inégal, de rétablir le lien entre les deux. Dans l'ancien système, on se confessait plus qu'on ne communiait et la confession était d'abord perçue comme une sorte de rituel de purification conditionnant l'accès à l'eucharistie. C'était l'un de ses principaux moteurs. «À telle personne, disait le dicton, on donnerait le Bon Dieu sans confession $»^{31}$, ce qui n'était pas le cas général. Le plus sûr était encore que l'intervalle entre les deux fût le plus bref possible: le scrupuleux qui se précipitait du confessionnal au banc de communion pour ne pas avoir le temps de pécher entre les deux était sans doute une exception, mais il ne faisait, en un sens, qu'exagérer une préoccupation considérée comme normale dans la culture spirituelle du temps.

Or, dans la période précédente, deux phénomènes avaient déjà commencé à modifier le système.

D'une part, la campagne en faveur de la communion fréquente, qui a pris un nouvel essor sous le pontificat de Pie X, amplifié par les mouvements apostoliques de l'entre-deux-guerres, notamment l'Action catholique. Elle s'est heurtée aux habitudes anciennes et aux résistances des hommes pour qui, même dans les régions les plus chrétiennes, la communion fréquente était considérée comme une pratique avant tout féminine ${ }^{32}$. Mais elle a tout de même fini par se répandre assez largement dans les années 1950, surtout chez les jeunes et les militants. En 1963 dans un texte célèbre, Gabriel Le Bras pouvait écrire: «[d]epuis le début de ce siècle, [...] la pratique sacramentelle a décuplé dans les élites populaires et bourgeoises ${ }^{33}$. Ce qui signifie - le point est d'importance - que la génération de la rupture est aussi, a priori, celle qui a les chances de s'être confessée

31. Comme le soulignait Pierre-Marie Gy, o.p., dans «Les bases modernes de la confession», dans La Maison-Dieu, 117, $1^{\text {er }}$ trimestre 1974, p. 73.

32. Voir la carte des «cénalisants» ruraux des années 1945-1965 dans l'Atlas de la pratique religieuse de Terrenoire et Isambert, et les commentaires de Claude Langlois.

33. Gabriel Le Bras, «Déchristianisation: mot fallacieux», dans Cahiers d'histoire publiés par les universités de Clermont-Lyon-Grenoble, t. IX, Grenoble, 1964, p. 97. 
le plus dans son enfance et sa jeunesse. Ceci expliquerait-il cela? La question mérite au moins d'être posée parce qu'elle attire notre attention sur le fait que la «crise catholique» a aussi été, de la part des «bons élèves» du catholicisme des années 1950, une réaction tardive contre une forme d'overdose religieuse. De fait, rarement christianisme aura été aussi «pur» et aussi peu «coupé» socialement et culturellement que celui de cette génération.

Le second élément qui a contribué à faire évoluer la pratique est l'assouplissement des règles du jeûne eucharistique au lendemain de la guerre. En 1947, une première série d'assouplissements avait été obtenue à la demande du cardinal Suhard ${ }^{34}$. En janvier 1953, Pie XII stipule, dans la constitution Christus Dominus, que la prise d'eau ne «détruit» plus le jeûne eucharistique et que celui-ci est réduit à une heure pour le liquide et trois pour le solide ${ }^{35}$. Les enfants des familles pieuses de l'après-guerre se souviennent encore aujourd'hui d'avoir planté leur cuillère dans leur bol du petit-déjeuner dominical pour savoir s'ils s'apprêtaient à manger de la nourriture solide ou liquide! Plus généralement, un nombre croissant de fidèles a pris l'habitude, après la guerre, de communier fréquemment en public, à la grand-messe, et non plus, comme c'était souvent le cas auparavant, lors d'une messe basse matinale, dite «de communion» (ce qui impliquait théoriquement de revenir pour la grand-messe ensuite), ou en dehors de toute messe, lors d'une distribution ad hoc.

Après le concile, dans le prolongement de ces évolutions antérieures, la communion s'est banalisée au point de faire désormais plus ou moins partie du rite lui-même, sauf peut-être pour les divorcés remariés qui sont à peu près les seuls à être restés attachés malgré eux aux normes de l'ancien système. L'historien Paul Vigneron, dans un ouvrage engagé de 1976 qui n'a pas perdu tout intérêt, a bien souligné le paradoxe: «les prêtres [sont rares] qui rappellent encore aux laïcs que le chrétien ne peut communier, s'il a commis des fautes graves contre la Loi de Dieu, qu'après s'en être confessé. Alors qu'ils manifestent beaucoup de sévérité pour l'admission à

34. Voir La documentation catholique, $\mathrm{n}^{\circ} 1006,21$ décembre 1947, col. 16011608.

35. Pie XII, Constitution apostolique Christus Dominus sur la discipline à observer à l'égard du jeûne eucharistique (6 janvier 1953), dans La documentation catholique, $\mathrm{n}^{\circ} 1139,25$ janvier 1953, col. 65-72. 
d'autres sacrements, semblant toujours redouter que la démarche des fidèles ne soit pas assez sincère, des prêtres les encouragent à communier "sans problème" $»^{36}$. L'idée s'est imposée de plus en plus qu'il était légitime de communier sans s'être confessé au préalable ${ }^{37}$.

\section{La lassitude de la jeune génération sacerdotale des années 1950-1960}

L'évolution de l'attitude du clergé n'est pas moins importante dans cette histoire car la confession - on l'oublie souvent - était une double contrainte: pour les fidèles, certes, mais aussi pour le clergé, réduit à passer de longues heures enfermé dans un confessionnal inconfortable à entendre des récits de vie monotones, dans lesquels la «qualité des accusations» faisait le plus souvent défaut. La confession, si elle pouvait être le théâtre d'échanges d'une grande intensité, voire de véritables conversions, qui comptaient parmi les moments les plus forts d'une vie sacerdotale, était le plus souvent un pensum dont le clergé se serait bien passé, contrairement à ce qu'une certaine littérature anticléricale a pu s'imaginer assez gratuitement. Les manuels de confesseurs d'après-guerre insistaient sur l'obligation qu'avait le clergé de ne pas se soustraire à ce devoir ou de l'abréger indûment, preuve qu'il en avait parfois la tentation. Et de donner généralement l'exemple du curé d'Ars qui n'hésitaient pas, au besoin, à passer dix-huit heures par jour au confessionnal.

L'enquête orale que j'ai menée auprès des pensionnaires de la maison de retraite du clergé du Landreau en Vendée a amplement confirmé le fait. Mes interlocuteurs m'ont dressé, non sans humour, un tableau sans nostalgie de la confession de masse des années 1950: les files de pénitents qu'on ne gardait généralement pas plus d'une minute par personne parce qu'autrement, on aurait pu les compromettre aux yeux de leurs voisins; qu'on accueillait en leur disant: «Pas de "Je confesse à Dieu", vos péchés tout de suite»; des confessions mécaniques reconduites ne varietur depuis l'enfance (les grands-mères s'accusant par exemple d'avoir volé de la confiture) ou décalquées d'un examen de conscience omnibus,

36. Paul Vigneron, Les crises du clergé français, 1976, p. 423-424.

37. Voir, par exemple, le point de vue de J. M. R. Tillard, o.p., dans «Pénitence et Eucharistie», dans La Maison-Dieu, 90, 2 trimestre 1967, p. 103-131. 
etc. Sans parler des confessions d'enfants, clientèle forcée des confessionnaux qui s'accusaient parfois de n'importe quoi et chez qui manquait surtout le plus souvent la matière première de l'opération, c'est-à-dire les péchés ${ }^{38}$.

Sans doute faut-il faire la part, dans l'interprétation de ces souvenirs, d'un certain nombre de biais possibles, comme le fait que la Vendée était une région de «chrétienté» qui a atteint assez tard (dans l'entre-deux-guerres) le sommet de son encadrement clérical; qu'on n'a plus affaire aujourd'hui, par la force des choses, qu'aux jeunes prêtres des années 1945-1960, et pas à leurs confrères plus âgés, qui avaient sans doute un avis différent sur le sujet; que l'événement-Vatican II a pour eux une dimension fortement identitaire et qu'il a certainement modifié la vision qu'ils avaient du catholicisme de leur jeunesse; que leur génération, enfin, est celle qui a assisté à l'effondrement de la confession et qu'elle peut de ce fait (même si ce n'est pas très sensible dans les témoignages) avoir la tentation de se justifier en arguant du fait que le gain qualitatif opéré compense amplement les pertes quantitatives.

Car on devine en les écoutant, derrière le grand récit de la «libération» conciliaire dont il n'y a pas de raison de mettre en doute la sincérité, ce qu'il pouvait y avoir aussi de satisfaction professionnelle et spirituelle dans le régime antérieur, au terme d'une journée éprouvante de confessions, à l'idée du devoir accompli et du rôle qu'on avait joué dans la «purification» de tout un «peuple» (à défaut d'échanges très personnalisés ou très intenses). Ceci doit nous sensibiliser à un aspect important du sujet, à savoir: le changement dans l'horizon d'attente du confesseur, devenu plus exigeant en termes de sincérité et d'intensité des échanges, à mesure que la religion cessait progressivement d'être un «fait de mentalité», collectif et indiscuté, pour devenir un «fait d'opinion», discutable et plus personnel (pour reprendre une distinction de

38. La pratique donnait lieu dans la littérature spécialisée à des passages assez comiques, comme celui-ci, tiré du manuel de Chanson: "Certains enfants s'accusent de tout ce qui est indiqué sur l'examen de conscience imprimé. Parfois, ils disent oui ou non au petit bonheur - ou même successivement. On peut alors leur demander s'ils ont commis la simonie? le suicide... et combien de fois !... Si l'enfant répond: oui et indique le nombre de fois, le C[onfesseur] sera fixé sur le crédit à apporter aux autres accusations.» (abbé A. Chanson, Pour mieux confesser, Arras, Brunet, 1949 ( $1^{\text {re }}$ édition 1948), non paginé, $\mathrm{n}^{\circ} 357$ ). 
Philippe Boutry $)^{39}$. Sans doute mes interlocuteurs ont-ils vécu dans leur carrière ecclésiastique un changement de ce type et ont-ils parfois tendance, du coup, à rétroprojeter dans l'évaluation du passé les critères du présent, acquis entre-temps.

\section{Le calendrier de la réforme et son caractère relativement tardif}

Le calendrier de la réforme constitue une autre raison probable du déclin, même si son effet est moins facile à cerner que les causes précédentes. Tous les gouvernants confrontés à la gestion d'une grande réforme connaissent ce type de problèmes: trop tôt, on n'en voit pas la nécessité, trop tard, on ne vous sait pas gré de ce qui apparaît comme un ralliement contraint à un fait acquis, trop vite, on traumatise les gens, et trop lentement, on laisse pourrir les situations. Ils n'ont, en un sens, que le choix des mauvaises solutions. La constitution conciliaire sur la liturgie, qui est la première à avoir été adoptée par le concile Vatican II, dès décembre 1963, se présentait comme l'aboutissement d'un mouvement liturgique plus ancien en même temps que comme un programme de réforme. Celle du sacrement de la pénitence était annoncée laconiquement, au milieu de beaucoup d'autres, dans le numéro soixante-douze de la constitution: «Le rite et les formules du sacrement de la pénitence seront révisés de telle sorte qu'ils expriment plus clairement la nature et l'effet du sacrement.» Déclaration prudente, d'où l'on pouvait déjà conclure a minima cependant que l'ancien rite manquait de «clarté», ce dont beaucoup ne s'étaient probablement pas avisés jusque-là.

Mais la réforme de la pénitence n'était pas la priorité et elle n'est intervenue qu'en 1974, avec la promulgation du nouveau rituel romain, le dernier de ses grands textes constitutifs ${ }^{40}$. La difficulté, dont ses concepteurs étaient parfaitement conscients, était que l'annonce même de la réforme avait pour effet, même si telle n'était pas son intention, de suspendre de facto le système en vigueur, considéré désormais comme provisoire, et donc plus ou moins caduc. Cette

39. Philippe Boutry, Prêtres et paroisses au pays du curé d'Ars, Paris, Le Cerf, 1986, passim.

40. Le nouvel Ordo paenitentiae datait du 2 décembre 1973 mais il n'a été rendu public que le 7 février 1974. Voir la «Note» en français qui accompagnait sa publication dans La Documentation catholique, t. LXXI, n 1649, 3 mars 1974, p. 209-210. 
difficulté était redoublée dans le cas de la pénitence par le fait que la réforme a été tardive. En attendant - mais il faudrait voir de plus près ce qu'il s'est passé exactement dans cette période intermédiaire les fidèles ne savaient plus très bien comment se confesser, ni même s'il était toujours nécessaire de le faire ${ }^{41}$. Y avait-il lieu, par exemple, de confesser des péchés comme l'abstention de la pratique dominicale ou la limitation des naissances (avant Humanae vitae en juillet 1968)? Le confessionnal, si décrié, était-il toujours bien le lieu normal de la confession? Quand devait-on se confesser, puisque le clergé, pour éviter la routine, a souvent voulu débanaliser les veilles de fêtes autrefois réservées à cet effet? Etc.

\section{Le problème de la contraception}

La raison suivante est plus connue: il s'agit de la question de la limitation des naissances et de la contraception. Certains ont expliqué qu'Humanae vitae avait été en 1968 la goutte d'eau qui avait fait déborder le vase et que Paul VI avait porté de cette manière un coup mortel à la confession ${ }^{42}$. Le cardinal Cottier, qui fut le théologien privé du pape Jean-Paul II et qu'on ne soupçonnera pas d'hostilité de principe à l'égard du texte, disait: «Depuis Humanae vitae, la crise du sacrement de pénitence a explosé $»^{43}$. La thèse comprend certainement une part de vérité. Mais jusqu'à quel point? Il est assez difficile de le dire.

Les grandes lignes de cette histoire sont relativement bien connues désormais grâce aux travaux de John Noonan, Martine Sevegrand et Claude Langlois ${ }^{44}$. La décision de Pie XI en décembre

41. Henri Baudry, Synthèse des réunions de quartiers (189). Canton de Saint Fulgent, 3000 participants, décembre 1970, 1971.

42. Guy Bechtel, La chair, le diable et le confesseur, Paris, Hachette, coll. «Pluriel», 1994. Bon exemple de «delumisme» historiographique (au mauvais sens du terme).

43. Cité par Martine Sevegrand dans L'affaire Humanae vitae, 2008, p. 146.

44. Voir notamment John T. Noonan, Contraception et mariage. Evolution ou contradiction dans la pensée chrétienne?, traduit de l'anglais par Marcelle Jossua, Paris, Le Cerf, 1969; Martine Sevegrand, Les enfants du bon Dieu. Les catholiques français et la procréation au XX siècle, Paris, Albin Michel, 1995, et L'affaire Humanae vitae. L'Eglise catholique et la contraception, Paris, Karthala, coll. «Disputatio», 2008; Claude Langlois, Le crime d'Onan. Le discours catholique sur la limitation des naissances (1816-1930), Paris, Les Belles Lettres, coll. «L'âne d'or», 2005. 
1930, par l'encyclique Casti connubii, de condamner la limitation artificielle des naissances et d'exiger des confesseurs qu'ils interrogent les pénitents sur ce point, sous peine de prévarication, a marqué une date importante ${ }^{45}$. Elle contrastait avec la tolérance qui avait prévalu jusqu'alors depuis le XIX $^{\mathrm{e}}$ siècle. Toute une génération de chrétiens a donc été vigoureusement «recadrée» dans ce domaine à partir des années 1930, même si Pie XII a apporté des tempéraments dans les années 1950, en approuvant la «régulation des naissances » (le birth control catholique) et en encourageant la diffusion des méthodes naturelles. Les autorités étaient formelles: il n'y avait aucune chance de voir évoluer la position officielle dans ce domaine, comme elle avait pu le faire, par exemple, dans celui des règles du jeûne eucharistique. Dans le manuel de Chanson, largement répandu dans les séminaires et presbytères des années $1950, l^{\prime}$ '«onanisme conjugal » faisait l'objet d'une partie entière sur les six que compte l'ouvrage.

Sur ce intervint le concile et les débuts de la commercialisation de la pilule. Ce qui avait paru indiscutable sous les pontificats précédents parut soudain, sinon remis en cause, du moins remis sur l'établi, même si Paul VI retira la discussion de la question au concile pour la confier à la commission pontificale spéciale créée à cet effet par son prédécesseur (tout comme celle du célibat ecclésiastique). Au printemps 1967, des fuites dans la presse anglosaxonne puis française ${ }^{46}$ révélèrent que la majorité de la commission était favorable à une évolution de la position catholique, au nom de l'«amour conjugal» et de la «paternité responsable», avant que Paul VI, en juillet 1968, ne réaffirme la position de Pie XI et Pie XII, sans beaucoup d'accommodement pastoral.

Cette gestion chaotique du dossier a pesé lourdement sur la crédibilité de l'institution. Elle a d'abord paru douter de la nécessité de maintenir les anciennes obligations, puisqu'elle a réuni une commission pour étudier la question, puis, après avoir longtemps hésité, au prix d'une dramatisation croissante des attentes, accrue

45. Je me permets de renvoyer ici à mon article: «Quelques éléments concernant l'encyclique Casti connubii (1930) sur le mariage chrétien», dans Pie XI et la France, sous la direction de Jacques Prévotat, École française de Rome, 2010, p. 347-367.

46. Contrôle des naissances et théologie. Le dossier de Rome, traduction, présentation et notes de Jean-Marie Paupert, Paris, Le Seuil, 1967. 
par les fuites de la presse, elle est finalement revenue à la position antérieure, perdant à la fois les bénéfices de l'évolution (puisqu'elle n'a pas eu lieu) et de l'immobilisme (puisqu'elle a paru hésiter). Difficile d'imaginer scénario plus catastrophique pour une institution.

Mais il ne faut sans doute pas s'exagérer malgré tout l'effet de l'encyclique sur la confession. D'abord parce que, chronologiquement, le décrochage massif est antérieur et remonte au milieu des années 1960. Ensuite parce que la crise a été moindre en France que dans les pays anglo-saxons et en Allemagne (le choc ayant été amorti par la prise de position de l'épiscopat). En novembre 1968, en effet, celui-ci a expliqué que ce «désordre» n'était pas toujours «coupable» et que la chasteté conjugale défendue par l'encyclique devait surtout être considérée comme un idéal ${ }^{47}$. Enfin parce que la France avait depuis longtemps (dès la fin du XIX ${ }^{\mathrm{e}}$ siècle) achevé sa transition démographique par la généralisation d'une contraception «mécanique» massivement répandue et que seuls les milieux les plus fervents ou les plus militants attachaient de l'importance au point de vue de l'Église dans ce domaine.

\section{La désaffection du confessionnal, symptôme et facteur de crise}

Une autre raison est liée à la désaffection du confessionnal. L'explication pourra paraître paradoxale parce que pour une partie du clergé et des fidèles son abandon a été vécu, au contraire, comme une libération. Mais c'était loin d'être le cas général.

Le code de droit canonique de 1917 précisait que «[1]es confessions ne seront pas entendues en dehors du confessionnal, à moins d'une juste cause $»^{48}$. En 1948, l'abbé Chanson rappelait encore que les femmes devaient être entendues au confessionnal, sauf «vraie nécessité» (vera necessitas) qu'il convenait de ne pas entendre trop largement, comme certains en avaient la tentation. C'était le cas notamment des aumôniers de mouvements de jeunes, qui inclinaient à lui préférer, à l'occasion d'un grand rassemblement ou d'un pèlerinage, la confession déambulatoire de plein air. Le contrôle du confessionnal faisait encore partie, dans les années

47. «Note pastorale de l'épiscopat français sur Humanae vitae», dans $L a$ documentation catholique, t. LXV, $\mathrm{n}^{\circ} 1529,1^{\text {er }}$ décembre 1968, col. 2055-2066.

48. Canon $964 \& 3$. 
1950, des passages obligés de la visite canonique de l'évêque dans les paroisses.

Car le confessionnal n'était pas seulement un avatar du problème de la confession des femmes, comme on a parfois eu tendance à le dire pour mieux le ridiculiser. Il avait surtout pour objectif d'éviter que la «fausse honte» ou le «respect humain» (comme on disait dans les manuels) ne tiennent les fidèles à distance de la confession, en préservant un certain anonymat. D'où l'attention prêtée à des données très concrètes, comme leur localisation dans les églises, leur visibilité (suffisante sans être ostentatoire), les facilités de circulation à préserver, la disposition des chaises alentour pour éviter les indiscrétions, l'affichage clair des horaires et des noms des confesseurs, etc. Il n'était pas jusqu'aux grilles ou «tirettes» des confessionnaux dont les trous devaient être suffisamment petits pour que confesseurs et pénitents ne se voient pas trop nettement ${ }^{49}$.

Or il est devenu, dans les années 1960, aux yeux d'un grand nombre de croyants, ce qu'il était depuis longtemps dans une partie de la littérature anticléricale: le symbole, mi-odieux, mi-ridicule, de l'ancien régime pénitentiel, celui avec lequel il fallait rompre absolument parce qu'il était synonyme de misogynie, d'individualisme, d'hypocrisie, de routine, de «surculpabilisation», etc. On n'en finirait pas de citer tous les textes qui illustrent ce procès d'époque du confessionnal, d'origine généralement cléricale ${ }^{50}$.

La publication du nouveau rituel de la pénitence en 1974 a marqué une étape importante dans l'amplification de sa désaffection. Il confiait aux conférences épiscopales le soin «d'établir avec plus de précision les règles sur le lieu approprié pour la célébration ordinaire de la Pénitence» ( $\left.n^{\circ} 38\right)$. Par ailleurs, il insistait sur la dimension

49. Voir par exemple: abbé A. Chanson, éd. 1949, n 335, n 339; Adrien Cance, p.s.s., Le Code de droit canonique. Commentaire succinct et pratique, t. 2, Paris, Lecoffre/Gabalda, 1951, p. 373-374, note 1.

50. Par exemple: «Quel confesseur ne se souvient d'avoir scruté dans la pénombre les intonations de la voix qui lui parvenaient par les interstices d'une grille plus souvent située au niveau de son coude qu'à celui de son oreille, pour tenter de situer à l'aveuglette son interlocuteur? d'avoir guetté la venue d'adjectifs, révélateurs parce que le genre s'en accorde avec celui du sujet, permettant au moins de savoir si on avait affaire à un homme ou à une femme? Non, du reste, qu'il faille brûler les vénérables meubles légués par l'Église post-tridentine et le $\mathrm{XIX}^{\mathrm{e}}$ siècle: pour certains pénitents, la discrétion qu'ils permettent fait partie de leur vérité humaine et mérité, de la part du confesseur, un entier respect. » (Etienne Cellier, s.j., «Au confessionnal», dans Christus, t. 25, nº 97, janvier 1978, p. 51). 
«dialoguée» (au sens d'échange spirituel et non plus simplement, comme autrefois, d'interrogatoire du pénitent par le prêtre) que devait revêtir la «bonne» confession. Mgr Bunigni, secrétaire de la Congrégation pour le Culte divin, dans le commentaire autorisé qu'il a donné du texte en février 1974, écrivait: «si le sacrement de la pénitence conféré individuellement doit conduire à un renouveau de vie, il doit se dérouler sous forme d'un dialogue serein et tranquille entre le prêtre et le pénitent, accompagné de prière et, lorsque cela est possible, de lecture de la Parole de Dieu, dans un cadre adapté, également du point de vue matériel et sans être pressé par le temps $»^{51}$. Le confessionnal n'était pas idéal pour ce type d'échanges, a fortiori s'il devait se terminer, comme il était prévu, par une imposition des mains.

Or un grand nombre de fidèles restaient attachés à la pratique traditionnelle et, dans certains cas (les souvenirs dans ce domaine ne manquent pas), il leur a fallu batailler ferme pour obtenir qu'on veuille bien leur en conserver provisoirement l'usage. Dans le dossier du Pèlerin de 1974, le prêtre et sociologue Julien Potel, pourtant favorable aux évolutions en cours, montrait bien que «se confesser avec un prêtre seul dans un confessionnal» restait la manière préférée de se confesser d'une majorité de pratiquants réguliers ( $41 \%$ contre $31 \%$ qui préféraient «se confesser seul avec un prêtre en dehors du confessionnal»). «À travers ces proportions élevées, concédait-il, s'expriment le désir de conserver un certain anonymat et le fait d'avoir toujours fait ainsi jusqu'à une époque récente $»^{52}$. À telle enseigne que, face à cette situation, l'historien finit par se demander $q u i$ au juste souhaitait exactement sortir des confessionnaux et si leur disqualification très volontariste n'a pas été, dans la perspective du maintien des taux, le type même de la «fausse bonne idée» pastorale.

\section{L'effet paradoxal des «célébrations pénitentielles» avec absolution collective}

La publication en 1974 du nouveau rituel romain de la pénitence n'a pas enrayé la chute de la confession. En 1952, $37 \%$ des

51. Interview diffusée par Radio-Vatican le 7 février 1974, reprise dans $L a$ Documentation catholique, t. LXXI, nº 1649, 3 mars 1974, p. 211.

52. Le Pèlerin du $20^{e}$ siècle, 3 novembre 1974, «Les Français et la confession. Un sondage SOFRES-Pèlerin », p. 38. 
catholiques déclaraient ne jamais se confesser. En 1974, ils étaient $54 \%$ et en 1983, $69 \%$. Il est probable que l'aller et retour magistériel dans la question des «célébrations pénitentielles» avec absolution collective, en ajoutant à la confusion, a aggravé la crise.

Il faut revenir ici brièvement sur l'origine de ce débat qui reste, en l'absence d'archives et d'études approfondies, passablement obscure. La confession était restée après la guerre largement en dehors du mouvement de rénovation liturgique qui allait trouver sa consécration dans la constitution conciliaire Sacrosanctum concilium, même si elle avait donné lieu dans les décennies précédentes à d'importants travaux historiques et théologiques qui ont bien mis en évidence la diversité et la variabilité de ses formes passées. Sans doute ne voyait-on pas très bien à ce stade comment ce sacrement privé, voire secret, et quelque peu immatériel, pouvait s'insérer dans un mouvement qui insistait surtout sur le caractère communautaire et ecclésial de la liturgie.

À partir de la fin des années 1950 cependant, des essais de «confession communautaire» ont eu lieu. Il s'agissait concrètement de cérémonies pénitentielles collectives suivies de confessions individuelles, comme on en organisait parfois pour les enfants jugés incapables de faire tout seuls leur examen de conscience. La revue de liturgie La Maison-Dieu a publié en 1958 un premier schéma de ce type ${ }^{53}$. Le phénomène a commencé à se répandre au début des années 1960, dès avant le Concile ${ }^{54}$. Mais il n'était pas encore question à cette date de célébrations pénitentielles avec absolution collective (c'est-à-dire permettant de faire l'économie de la confession auriculaire).

L'absolution collective était une procédure prévue de longue date dans les manuels et les codes, mais réservée à des cas tout à fait exceptionnels, liés généralement à un péril de mort imminent et à des situations de guerre. Il est vrai que la Première et la Seconde Guerre mondiale les avaient multipliés, si bien qu'une instruction de la Sacrée Pénitencerie du 25 mars 1944 avait dû rappeler que

53. Robert Meurice, f. c., «Les célébrations de la pénitence, suggestions et expériences », dans La Maison-Dieu, 56 (4 trimestre 1958), p. 76-95.

54. Voir les références données par Jean-Pierre Jossua, D. Duliscouët, Bernard Marliangeas dans leur «Bulletin de théologie. Crise et redécouverte du sacrement de pénitence», dans Revue des sciences philosophiques et théologiques 52, 1968, p. 119-142. 
toute absolution collective donnée en dehors de ces situations exceptionnelles constituait un abus grave.

Il faut attendre la fin des années 1960 pour que l'on commence à en parler ${ }^{55}$, sans que l'on sache très bien où les premiers essais ont été accomplis. En juin 1972 cependant, le phénomène est déjà suffisamment répandu pour que la Congrégation pour la doctrine de la foi juge nécessaire de rappeler que la confession auriculaire devait rester la voie ordinaire de la pénitence, ce qu'elle avait manifestement déjà cessé d'être pour certains. «Il faut, disait-elle [...] réprouver la pratique qui s'est introduite récemment çà et là, par laquelle on prétend satisfaire au précepte de la confession sacramentelle des péchés mortels en vue d'obtenir l'absolution par la seule confession générale ou accomplie, comme on dit, de façon communautaire. $»^{56}$

En 1974 cependant, le nouveau rituel, tout en rappelant que la confession auriculaire devait rester la forme ordinaire du sacrement, présentait les célébrations pénitentielles avec absolution collective comme l'une des trois formes possibles de la pénitence. Si certains ont pu regretter qu'on n'ait pas supprimé tout bonnement la confession ou donné à l'acte pénitentiel du début de la messe un caractère proprement sacramentel, beaucoup dans les milieux «avancés» se sont félicités de voir que ces cérémonies avaient désormais leur rite et qu'elles étaient peu ou prou mises sur le même pied que les autres formes de la pénitence (confession classique et célébrations pénitentielles suivies d'absolutions individuelles) ${ }^{57}$.

Ce type de cérémonies s'est multiplié à partir de 1974 et elles ont, de fait, rencontré un succès certain, au moins dans un premier temps. Le théologien d'origine vendéenne Louis-Marie Chauvet le soulignait en 1976, non sans identifier au passage le principal motif de leur succès: "pour de plus en plus de chrétiens, de tout niveau et de tout milieu, l'aveu individuel des péchés à un prêtre représente aujourd'hui, de fait, un obstacle majeur à leur participation au

55. Par exemple: Walter Kasper, «Confession hors du confessionnal?», dans Concilium, $\mathrm{n}^{\circ} 24,1967$, p. 35-44.

56. Congrégation pour la Doctrine de la foi, «Normes pastorales pour l'administration de l'absolution sacramentelle générale», dans La Documentation catholique, 6-20 août 1972, t. LXIX, nº 1614, p. 713.

57. Par exemple: Pierre Jounel, «La liturgie de la réconciliation», dans $L a$ Maison-Dieu, 117, $1^{\text {er }}$ trimestre 1974, p. 7-37, notamment p. 36. 
sacrement de la réconciliation $»^{58}$. Au début des années 1980 cependant, sous le pontificat de Jean-Paul II, Rome s'est efforcée d'en raréfier l'usage au maximum et de lui redonner de fait, non plus seulement de droit, ce statut de confession extraordinaire qu'elle avait jusque-là. C'était le sens du discours de Jean-Paul II aux évêques de la région Est en $1982^{59}$, du canon 961 du nouveau Code de droit canonique de janvier $1983^{60}$ et, avec des nuances, du rapport sur le sujet de la Commission théologique internationale de la même année ${ }^{61}$.

L'effet, en termes pastoraux, a été assez négatif non seulement parce que cette suppression s'est heurtée à un effet-cliquet difficile à remettre en cause mais parce que, dans la mesure où les fidèles avaient été attirés précisément par le fait qu'il s'agissait d'une forme de pénitence sans confession, il était peu probable de les voir revenir au système antérieur, une fois la fenêtre refermée ${ }^{62}$. $\mathrm{Au}$ contraire, tout donne à penser qu'ici aussi l'aller et retour magistériel a fonctionné pour beaucoup comme un sas de sortie de la confession traditionnelle.

\section{LES SIGNIFICATIONS DE LA CRISE}

Reste, pour finir, le problème de l'interprétation de la crise. Il est de bonne méthode, en pareille matière, de commencer par écouter

58. Louis-Marie Chauvet, «Réflexions au sujet de l'aveu dans le sacrement de la réconciliation», dans Église de Luçon, 20 mars 1976, 6, p. 91.

59. Jean-Paul II, «Discours aux évêques de la région Est», dans $L a$ documentation catholique, 2 mai 1982, $\mathrm{n}^{\circ} 1829$, p. 438-441.

60. L'absolution collective devait être réservée à des situations exceptionnelles, pas même aux rassemblements religieux. De la copia confessorum (abondance des fidèles à confesser) susceptible de la justifier, désormais seuls les évêques pouvaient décider.

61. «La réconciliation et la pénitence», dans La documentation catholique, 18 décembre 1983, n²2, p. 1158-1169.

62. Comme l'écrit Hubert Barbier, ancien évêque de Bourges: «dans ce contexte [de désaffection du sacrement] est arrivée la déclaration, maladroite, de Rome, très vite reprise par Rome, mais c'était fait, concernant les diverses formes possibles de la célébration de la réconciliation, déclaration qui semblait mettre comme une forme parmi les autres la célébration communautaire avec absolution collective, forme qui, précisément, dispensait d'un aveu et de répondre à l'aveu, deux actes devenus difficiles. [...] quand on entrouvre une telle porte, les fidèles poussent la porte et s'y engouffrent (les prêtres aussi, du reste).» (lettre à l'auteur de 2014.) 
ce qu'en ont dit les contemporains eux-mêmes, acteurs et témoins de la crise, qui n'étaient, après tout, pas si mal placés pour en juger.

Les défenseurs de l'ancien système avaient tendance à considérer que la chute de la confession était un indice capital du fait que les changements en cours depuis les années 1960, dans l'Église comme dans la société, avaient pris une mauvaise direction. La crise leur paraissait, dans ses motivations profondes, plus spirituelle que sociologique. Déjà Pascal, dans les hautes eaux religieuses du $\mathrm{XVII}^{\mathrm{e}}$ siècle, écrivait que les hommes détestaient à ce point la vérité qu'ils accueilleraient avec gratitude quiconque les délivrerait de l'obligation d'avoir à dire une fois par an et dans le secret qui ils étaient vraiment à leur confesseur. La pénitence serait toujours un «baptême laborieux» (pour reprendre la formule du concile de Trente) qui avait peu de chances de jamais séduire les fidèles. Quoi qu'on fasse ou qu'on dise, on ne ferait pas l'économie des conditions impératives de la pénitence réelle: pas d'absolution sans humilité, pas d'humilité vraie sans humiliation, et pas d'humiliation sans «confusion», c'est-à-dire, d'une manière ou d'une autre, sans perdre la face ${ }^{63}$. À ce compte-là, mieux valait peut-être en effet la pénombre du confessionnal...

Telle était encore, à peu de chose près, la position du jésuite François Varillon en $1968^{64}$. Dans un texte marquant sur la «facilité spirituelle», il soulignait que celle-ci était une tentation permanente dans la vie du chrétien mais qu'elle était particulièrement à craindre dans les périodes de mutation culturelle et religieuse, comme celle que l'on était en train de vivre, parce qu'il devenait plus difficile alors de faire le départ entre ce qui était effectivement et légitimement caduc dans les formes historiques du christianisme, et ce qui relevait de ses valeurs pérennes, et donc non négociables. Derrière la protestation contre la confession auriculaire, il croyait discerner un accès d'impénitence collective, c'est-à-dire le désir secret d'être soulagé d'une partie des exigences du christianisme lui-même.

Les partisans de la réforme du système avaient évidemment un tout autre point de vue. L'effondrement de la confession de

63. «Il n'y a pas de salut sans libération, pas de libération sans aveu, et pas d'aveu sans qu'on s'écroule.» (Bernard Bro, Le secret de la confession, 1983, p. 45.)

64. François Varillon, «Sur la tentation de facilité», dans Christus, $n^{\circ} 60$, t. 15 , octobre 1968 , p. $499-508$. 
masse des années 1950 les laissait sans nostalgie et leur paraissait constituer rétrospectivement la meilleure preuve de sa pauvreté spirituelle par-delà l'impression de force qui se dégageait hier encore de sa large fréquentation. Elle n'avait pas tenu le choc des bouleversements sociaux et ecclésiaux parce qu'elle n'avait pas de racines profondes. La «redécouverte» conciliaire du sens de la pénitence leur paraissait compenser amplement les effets d'une crise perçue comme avant tout sociologique et culturelle. Ils plaidaient généralement pour l'adoption d'un système pluriel dans lequel les «célébrations pénitentielles» constitueraient désormais le régime ordinaire de la pénitence et la confession individuelle ne serait plus réservée, dans l'hypothèse où elle serait maintenue, qu'à des cas ou des moments exceptionnels de l'existence.

Sur le fond, ils insistaient sur l'historicité de la pénitence ${ }^{65}$. Une histoire de mieux en mieux connue révélait l'ampleur de ces variations antérieures: l'institutionnalisation de la pénitence publique au $\mathrm{II}^{\mathrm{e}}$ siècle, le fonctionnement du système de la pénitence unique et publique aux $\mathrm{III}^{\mathrm{e}}-\mathrm{VI}^{\mathrm{e}}$ siècles, le passage à la pénitence privée et réitérable aux $\mathrm{VI}^{\mathrm{e}}-\mathrm{X}^{\mathrm{e}}$ siècles, sa généralisation après Latran IV, la poussée rigoriste du second $\mathrm{XVII}^{\mathrm{e}}$ siècle, la révolution liguorienne au XIX ${ }^{\mathrm{e}}$ siècle, etc. Sa plasticité passée était la caution de sa plasticité future et le moment paraissait venu de faire connaître à la pénitence l'une de ces révolutions nécessaires, analogue à celle qu'elle avait connue aux $\mathrm{VI}^{\mathrm{e}}-\mathrm{VII}^{\mathrm{e}}$ siècles, quand le régime de la pénitence publique était devenu impossible et qu'il avait fallu lui substituer celui de la pénitence privée.

Sans prétendre trancher ce débat théologique et pastoral dans lequel les deux parties me paraissent avoir mis le doigt, à travers leur prisme particulier, sur des aspects réels du problème, $\mathrm{j}$ 'avancerais, pour ma part et pour finir, trois hypothèses complémentaires.

La première, d'inspiration plus ou moins delumienne, part du constat que le sacrement de pénitence était depuis Latran IV la source de difficultés intrinsèques redoutables, liées à la nécessité d'avouer tous ses péchés, y compris sexuels, au prêtre de sa paroisse, dans une situation structurellement dissymétrique. Celle-ci n'a jamais

65. Voir la synthèse bibliographique de ces travaux dans Jean-Pierre Jossua, D. Duliscouët, Bernard Marliangeas, «Bulletin de théologie. Crise et redécouverte du sacrement de pénitence», dans Revue des sciences philosophiques et théologiques 52, 1968, p. 119-142. 
pu être totalement compensée par les garanties qu'il comportait et les aménagements qu'on a pu lui apporter, comme le secret de la confession, la mécanisation de l'aveu, le confessionnal, la possibilité de se confesser à l'extérieur de la paroisse, la certitude de l'absolution, etc. La confession a donc probablement toujours été, pour le commun des fidèles, une contrainte et parfois un problème: le plus coûteux, assurément, des «devoirs» du chrétien. Dans ces conditions, elle ne pouvait s'imposer au grand nombre que moyennant des habitudes, des contraintes (légales, canoniques, morales) et des motivations spirituelles (comme la crainte de l'enfer) capables d'emporter ces résistances. À partir du moment où les unes et les autres ont paru donner des signes de faiblesse, le système a lâché, aussi brutalement que logiquement.

Deuxième interprétation possible : la confession a été la victime à plus court terme d'une offensive culturelle généralisée contre toutes les formes de culpabilité et de culpabilisation qui s'est déployée très largement dans les années 1960-1970. La religion, qui n'était pas seule en cause, a constitué pour elle un terrain privilégié d'opérations, au prix d'une double réduction de la question de la culpabilité à celle de ses formes religieuses et de celles-ci à la seule confession. Le phénomène s'est développé comme une véritable campagne de dératisation dans la psychologie contemporaine, d'inspiration plus ou moins psychanalytique, dans le prolongement du débat d'après-guerre sur «l'univers morbide de la faute». La mise en cause des formes pathologiques de la culpabilité ou de la «surculpabilisation» dont le clergé se serait rendu coupable par le passé a fini par affecter le concept même de la culpabilité au sens religieux du terme (comme prise de conscience du péché). Le succès des thèses de Delumeau sur la «pastorale de la peur» dans les années 1970-1980 allait dans le même sens ${ }^{66}$. La psychanalyse - c'est un lieu commun que de le rappeler - a récupéré dans les années 19601970 une partie de l'ancienne clientèle des confessionnaux et de la direction de conscience, avant d'être victime à son tour d'un retournement ultérieur de conjoncture, au nom parfois d'arguments assez semblables à ceux qui avaient servi par le passé contre la confession (utilité psychologique limitée, culpabilisation, etc.).

66. Voir Guillaume Cuchet, Faire de l'histoire religieuse dans une société sortie de la religion, Paris, Publications de la Sorbonne, 2013, p. 141-164. 
Dernière interprétation possible: la confession, dans sa définition courante, était avant tout le moyen de laver son «âme» de la souillure $\mathrm{du}$ «péché mortel» afin d'être trouvé digne, au jour de la «mort» et du «jugement», d'échapper à l'«enfer» et de rejoindre le «ciel» pour une «éternité» de bonheur partagé. Or cette infrastructure théologique et spirituelle, liée à une pastorale des «fins dernières » qui fut longtemps très dramatisée dans l'Église (même si elle l'était déjà beaucoup moins dans les années 1950), a complètement disparu du discours ecclésial dans la décennie suivante, comme si elle était soudain devenue incroyable pour les fidèles et impensable pour les théologiens eux-mêmes. Ses principaux concepts dorment depuis dans les catéchismes et les traités de théologie dans l'attente d'éventuelles résurrections ${ }^{67}$. Les historiens des mentalités (du «purgatoire» de Le Goff et Vovelle à la «mort» d'Ariès), qui ne sont jamais pour les croyances qu'ils étudient des oiseaux de très bon augure, s'en sont emparés dans les années 1960-1970, comme pour leur donner le coup de grâce. Ce n'est pas le lieu d'analyser ici les causes de ce phénomène fondamental, qui tient à la fois de l'aboutissement de processus d'érosion des croyances au long cours, du sacrifice théologique consenti au monde moderne pour sceller le pacte de la réconciliation avec lui et de l'accès de rousseauisme collectif. Mais le fait est que la confession a perdu dans l'opération une bonne partie de ses justifications traditionnelles, sans avoir retrouvé depuis l'équivalent.

La crise des années 1960-1970, outre le fait qu'un certain nombre de catholiques ont continué, malgré tout, de se confesser depuis, ne marque sans doute pas pour la confession la fin de l'histoire. On a signalé en introduction que des signes donnent même à penser que se cherche actuellement dans le monde catholique le moyen d'une redécouverte du sacrement de la pénitence et de la réconciliation.

67. Même si certaines ne manquent pas de talent. On peut citer en ce sens deux tentatives de «décongélation» philosophique récentes. Côté laïque : certaines analyses de Giorgio Agamben qui circule dans la tradition théologique comme dans une carrière à concepts étranges et suggestifs. Voir notamment ses développements sur les «corps glorieux» dans Nudités, Rivages poche / Petite Bibliothèque, 2012, p. 129-145. Côté religieux : les ouvrages du philosophe Fabrice Hadjaj, notamment Réussir sa mort. Anti-méthode pour vivre, Paris, Presses de la Renaissance, 2005, et Le paradis à la porte. Essai sur une joie qui dérange, Paris, Le Seuil, 2011 (rééd. coll. «Points» en 2014). 
L'avenir dira ce qu'il en sera, mais la disparition progressive de ceux qui ont été formés dans l'ancien système pénitentiel et qui en ont rarement gardé de bons souvenirs, jointe à la montée de jeunes générations que les sociologues actuels s'accordent à décrire comme plus classiques ou plus conservatrices que leurs aînés, pourraient bien constituer des facteurs favorables. En attendant, il y aurait intérêt à développer dans ce domaine des comparaisons internationales pour évaluer la spécificité du cas français et procéder, pendant qu'il est encore temps, à des enquêtes orales auprès de ceux qui ont connu de l'intérieur l'ancien régime de la confession, à titre de pénitents ou de confesseurs. Les maisons de retraite du clergé sont, à l'instar de celle du Landreau en Vendée que nous avons fréquentée pour l'occasion, pleines de vieux pasteurs qui finissent leur existence modestement en mesurant du regard l'ampleur de l'évolution qui les sépare du monde de leur enfance ou de leur jeunesse. Il serait dommage de laisser cette expérience et ce savoir disparaître sans avoir pris le temps de le recueillir: qui sait ce qu'il pourrait nous apprendre de notre monde et, peut-être, pourquoi pas, de nous-mêmes ${ }^{68}$ ?

guillaume.cuchet@u-pec.fr

68. Cet article a bénéficié des échanges qui ont eu lieu au sein du séminaire sur l'histoire des formes de la pénitence depuis la Révolution que Philippe Boutry et moi-même avons animé en 2013-2014 au Centre d'anthropologie religieuse européenne (CARE) de l'EHESS. 